\title{
Is rare chromatin agglutination on chromosomes mutually related to metastasis? An hypothesis
}

\section{Hit Kishore Goswami*}

Retired Professor of Genetics, 24, Kaushal nagar, P.O. Misrod, Bhopal (MP) 462047, India

This brief communication mentionstwo rare chromosomal aberrations, the first one is well known since 1986 as marker dots, released from chromosome under some molecular influence and the other one is "chromatin agglutination on all chromosomes". This aberration is totally new observation as far as known to the author. An hypothesis appears evident that since this chromatin agglutination was associated with accelerated metastasis, may be this molecular mechanism has cause or consequence relationship with metastasis. Hypothesis needs examination by modern cancer cytogeneticists.

Studies on genotoxic assessments by lymphocyte cultures on persons exposed to Methyl Isocyanateg as in Bhopal during midnight of $2^{\text {nd }}-3^{\text {rd }}$ Dec 1984 (Union Carbide Tragedy) by our laboratory as well as by others have been well known for more than three decades. Our studies by standard lymphocyte cultures and staining with Giemsa, G and $\mathrm{C}$ banding and Feulgen's approaches on more than 600 persons (including MIC affected, family members and suitable controls, various syndromes and cancer patients etc). More than 300 persons were followed up for manifold studies for more than two decades. Comparative observationshad established that chromosomal damages have been installed among seriously exposed persons. Since studies were based on standard protocols of cell cultures and chromosome studies with specific staining schedules [1-12] our repetitions were also carried out on all schedules of simple G staining, G \& C banding and Feulgen's approaches. Besides observing persistent chromosomal aberrations among most seriously affected surviving persons for more than two decades we had categorically introduced a new nomenclature for thedefinite origin ofchromatin dots of variable sizes, named as "Marker Dots (MDs). These marker dots arethe outcome of some triggered molecular mechanism operative on chromatin. We had published clear pictures of chromosomes, observed in many cases revealing direct detachment of these dots [1-12] which had varied in origin from different chromosomes. These marker dots were associated with many pathological features more commonly with malignancies and recurrent abortions.

This note further refers to another very rare observation in the cells of lymphocytes obtained from a patient who according to clinicians had had multiple complications including initial onset of bone cancer. The patient had shown many polyploid cells (Figure A; chromosomes ranged from 76- 88 chromosomes);marker dotsand translocations involving two or even three chromosomes, were very frequent. But there were certain cells showing (Figure B) "chromatin agglutination" exactly corresponding to $\mathrm{G}$ bands on each chromosome. After a few months the patient died and clinicians could only tell that speeding of metastasis was highly pronounced. This is very difficult to say that whether the molecular genetic mechanism resulting in

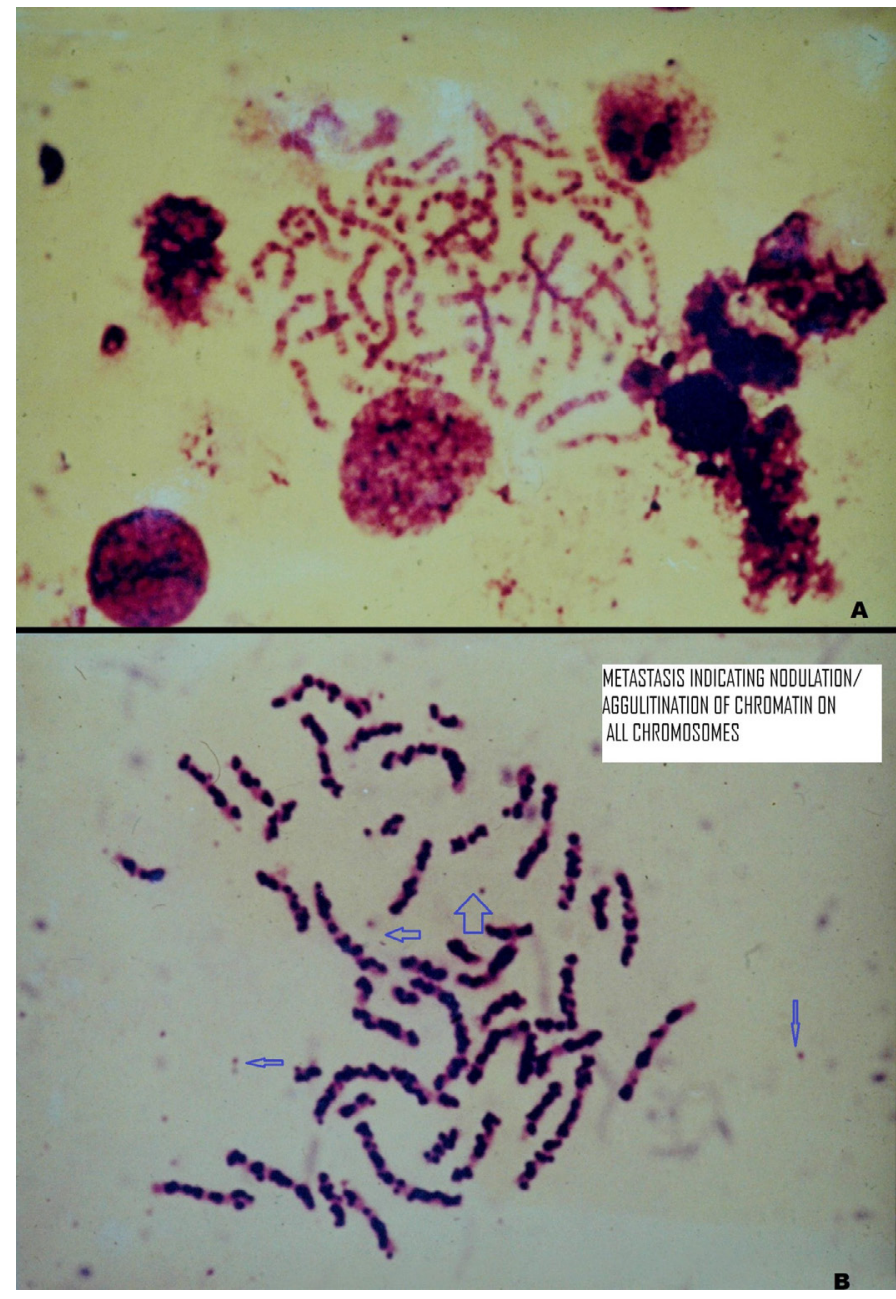

Figure 1. The lymphocyte culture from a woman exposed to methyl Isocyanate gas exposure in Bhopal during $2^{\text {nd }}-3^{\text {rd }}$ Dec in 1984

A) A G banded Polyploid cell showing thrown away some dots of chromatin

B) $\mathrm{G}$ banded but each band exhibits chromatin agglutination on all chromosomes. Many Marker dots are arrowed

${ }^{\star}$ Correspondence to: Hit Kishore Goswami, Retired Professor of Genetics, 24, Kaushal nagar, P.O. Misrod, Bhopal (MP) 462047, India, E-mail: hitkishoreg@gmail.com

Received: December 08, 2019; Accepted: March 30, 2020; Published: April 02, 2020 
chromatin agglutination was responsible for accelerated metastasis or metastasis had had induced chromatin agglutination. The cause and or consequence relationship in between these two needs to be examined by those laboratories which can venture taking a sample of blood for lymphocyte cultures from well diagnosed patients showing metastasis of any malignancy. Quite likely metastasis at the final peak my disrupt entire chromatinand unpack all chromosomes (?). This aberration is totally new observation as far as known to the author. We had examined more than 50 thousand metaphases from various persons which also included many abnormalities (13) and cancer patients during 1984-2002 but, had come across only two persons who have had revealed a few such metaphases. Both the patients were females and exhibited polyploid cells with multiple abnormalities. This must be mentioned that for chromatin agglutination, the MIC exposure cannot be blamed because such a situation was never observed. Such a stage of chromosomes can be indirectly associated with multiple cancer including bone cancer. Unfortunately, no opportunity ever existed for any repetition.An hypothesis appears evident that since this chromatin agglutination was associated with accelerated metastasis, may be this molecular mechanism has cause or consequence relationship with metastasis. Hypothesis needs examination by modern cancer cytogeneticists.

\section{Acknowledgements}

I am grateful to galaxy of my students who did work with me on human chromosomes during 1984-2002 in the department of Genetics, Barkatullah University Bhopal, India. I have frequently used slides and photographs for publications and I must confess, as usual, that I owe a great admiration for their very hard collaboration and sincere efforts.

\section{References}

1. GoswamiHK (1986) Cytogenetic effects of Methyl Isocyanate exposure in Bhopal. Human Genetics (Berlin) 74: 81-84. [Crossref]

2. Goswami HK, Chandorkar MS, Bhattacharya K, Vaidyanath G, Parmar D, et al (1990)Search for Chromosomal variations among gas exposed persons in Bhopal. HumanGenetics 84: 172-176. [Crossref]

3. Goswami HK, Rangnekar GV, Varshney S, Gandhi P, Jain B, et al. (1992) Crossed Renal Ectopia with pelvic lipomatosis: A new Syndrome involvingChromosome 1. Human Genetics 89: 666-670. [Crossref]

4. Goswami HK (1993) Marker dot is indicator of Chromosomal Mutagenesis. In: Haldane \& Evolution. Bionature 13(2): 325-333.

5. Goswami HK, Shrivastava N, Gopal SK, Sharma S, Chandorkar MS, et al. (1997) Unusual chromosomal features in a child with gradual disappearance of Right Ulna (mono ostolicosteolysis). JGenet Med 1: 11-16.

6. Goswami HK, Rangnekar AG, Sharma S, Varshney S, Lee IH, et al. (1998) Hemihypertrophy with hypomelanosis of Ito : A new syndrome combination. $J$ Genet Med. 2: 1-5.

7. Goswami HK, Chang SI (2001) Marker dots are expelled by attenuation in heterochromatin of a chromatid. Bionature 21: 41-48.

8. Goswami HK (2001) Genetic significance of marker dots.In, Perspectives in Cytology \& Genetics; Edts G K Manna \& S C Roy, AICC, Kalyani, India.

9. Goswami HK, Rangnekar A, Sikarwar LS (2011) Population Cytogeneticsof Abortions and Vanishing Twin. Indian Journal of Fundamental and Applied Life 1:2231-6345.

10. Goswami HK (2016) Cells with chromosomal aberrations trigger neoplastic transformations in humans. Twin Research and Human Genetics 19: 77-79.[Crossref]

11. Goswami HK (2017a) Is tendency to recurrently abort, a rejected malignant activity in developing embryonic tissue? ObstetGynecol Rep 1(2): 2-3.

12. Goswami HK (2017b) Gross chromosomal aberrations are early alarms for malignancy: A re-emphasis. Hereditary Genet 6: 187.

13. Goswami HK (2020) Many developmental errors and or pathological conditions may be associated with marker dots /small supernumerary chromosomes. J Family Med Community Health 7(1):1169.

Copyright: $\odot 2020$ Goswami HK. This is an open-access article distributed under the terms of the Creative Commons Attribution License, which permits unrestricted use, distribution, and reproduction in any medium, provided the original author and source are credited. 